\title{
Urate Transporter 1 Protein Levels and Localization in Type 2 Diabetic and Non-Diabetic Zucker Rat Kidneys
}

\author{
Yuriy Slyvka ${ }^{1}$, Felicia V. Nowak ${ }^{1}$, William Matthew Wooten $^{1}$, Kelly D. McCall ${ }^{2}$, Ramiro Malgor ${ }^{1}$, \\ Aaron S. Wood ${ }^{1}$, Sharon R. Inman ${ }^{1}$ \\ ${ }^{1}$ Department of Biomedical Sciences, Ohio University, Athens, USA \\ ${ }^{2}$ Department of Specialty Medicine, Ohio University, Athens, USA \\ Email: inmans@ohio.edu
}

Received December 9, 2012; revised January 9, 2013; accepted February 9, 2013

\begin{abstract}
Objective: Persons with type 2 diabetes have increased incidence of hyperuricemia and gout. The hypothesis that Urate transporter 1 (URAT1) levels are increased in type 2 diabetic Zucker rats and this is responsible for elevation of uric acid was tested. Methods: Male 12-week-old obese Zucker rats were compared to non-diabetic lean counterparts. Plasma glucose, uric acid and creatinine were measured. URAT1 protein levels in the renal cortex and medulla were determined by Western blot. Immunohistochemistry was used to determine the location of URAT1 in renal tubules. Results: Plasma glucose and uric acid levels were higher in the diabetic rats. There was no difference in plasma createnine. URAT1 antibody-positive bands of 27, 31, 50, 62 and $70 \mathrm{kDa}$ were observed in cortex. A similar pattern was observed in medulla with addition of a $44 \mathrm{kDa}$ band. No differences were observed in URAT1 proteins in the cortex between obese and lean rats. In the medulla, expression of the 44 and $50 \mathrm{kDa}$ proteins was higher in lean rats. Expression of 27, 50, $62 \mathrm{kDa}$ URAT1 proteins in the cortex was higher than in the medulla, while expression of the $70 \mathrm{kDa}$ URAT1 was higher in medulla than in cortex. Localization of URAT1 did not differ between groups and included tubules in both cortex and medulla. Conclusions: In male Zucker rats, URAT1 protein expression was observed in both kidney cortex and medulla. Uric acid elevation in the obese group was associated with decreases in the 44 and 50 kDa URAT1 proteins in renal medulla.
\end{abstract}

Keywords: URAT1 Transporter; Uric Acid; Zucker Rat; Type 2 Diabetes; Gout

\section{Introduction}

Although the body requires certain levels of uric acid (UA) because of its protective antioxidant and iron chelating properties [1,2], elevations of systemic UA can lead to gout [3]. Gout is the most common cause of inflammatory arthritis and currently affects $4 \%$ of the adult population in the United States ( 8.3 million people) [4]. The incidence of gout is much higher in persons with type 2 diabetes mellitus (T2DM) than in non-diabetics [5]. Diabetic nephropathy is associated with elevated serum UA levels [6], and there is increasing evidence that gout, itself, increases the risk for diabetes, kidney disease, obesity and hypertension [7]. Patients with both gout and T2DM share common genetic factors and there is a mutual interdependent effect on higher incidences [5]. URAT1 (Slc22 A12) and URATv1 (Glut9, Slc2A9) play a major role in UA kidney excretion in humans, rats and mice [8,9]. URAT1 is found in the renal tubular system and is responsible for the reabsorption of UA $[3,8]$. A number of other transporters including OAT 1 (Slc22A6), 3 (Slc22A8), 10 (Slc22A13), and 4 (Slc22A11), NPT1
(Slc17A1), NPT4 (Slc17A3), UAT/galectin9 and uromodulin contribute to urate transport in the kidney [10-13]. Increased protein levels of URAT1 are observed in mice with obesity/metabolic syndrome [14], and insulin resistance enhances urate reabsorption by up-regulating URAT1 expression in rats fed a high purine diet [15]. The hypothesis that URAT1 is up-regulated in the kidney in T2DM was tested in the obese Zucker rat (fa/fa) model of T2DM [16-18].

\section{Materials and Methods}

\subsection{Animal Model}

Male obese (fa/fa) Zucker rats $(\mathrm{n}=9)$ and lean Zucker rats $(n=9)$ were obtained from Harlan-Sprague Dawley (Indianapolis, IN), housed under controlled conditions of lighting, temperature and humidity, and fed regular chow. Males were chosen because they have higher levels of circulating UA than females [19,20]. At 12 weeks of age, animals were euthanized and blood and kidneys removed for analysis. All animal procedures were approved by the 
Ohio University Institutional Animal Care and Use Committee.

\subsection{Glucose, Creatinine and Uric Acid Levels}

Fasting blood glucose was measured using the One Touch Lifescan glucometer (Johnson and Johnson, New Brunswick, NJ). Plasma UA (QuantiChrom ${ }^{\mathrm{TM}}$ Uric Acid Assay Kit, BioAssays Systems, Hayward, CA) and createnine (QuantiChrom ${ }^{\mathrm{TM}}$ Creatinine Assay Kit, BioAssays Systems, Hayward, CA) were also quantified.

\subsection{Western Blot Analysis of URAT1 Levels in Renal Cortex and Medulla}

Immunoblots were done to assess URAT1 protein levels in kidney cortex and medulla. The right kidney was separated into cortex and medulla and homogenized in lysis buffer [10 mM Tris-HCl (pH 7.5), $150 \mathrm{mM} \mathrm{NaCl,} \mathrm{1 \%}$ Nonidet P-40] containing protease inhibitors. Thirty micrograms of protein was then subjected to SDS-PAGE and Western blotting using the Nu-PAGE System (Invitrogen, Grand Island, NY). Blots were probed with antiURAT1 (Abbiotec, LCC \#250521, San Diego, CA), stripped, and re-probed with anti- $\beta$-actin (Cell Signaling Technology, \#4967, Danvers, MA). Signals were visualized and bands quantified using the LI-COR Odyssey Infrared Imaging System (LI-COR, Lincoln, NE). Results are presented as URAT- $1 / \beta$-actin density ratio.

\subsection{Immunohistochemistry (IHC)}

Following rapid removal, half of the remaining kidney was fixed, embedded and processed as previously described [21]. Sections were incubated overnight at $4^{\circ} \mathrm{C}$ with primary rabbit polyclonal URAT1 antibody (3 $\mu \mathrm{g} / \mathrm{ml}$, Abbiotec, LCC \#250521, San Diego, CA). Isotype controls were performed on a consecutive section using isotype normal rabbit IgG at equivalent concentration to the primary antibody (Invitrogen Corporation,

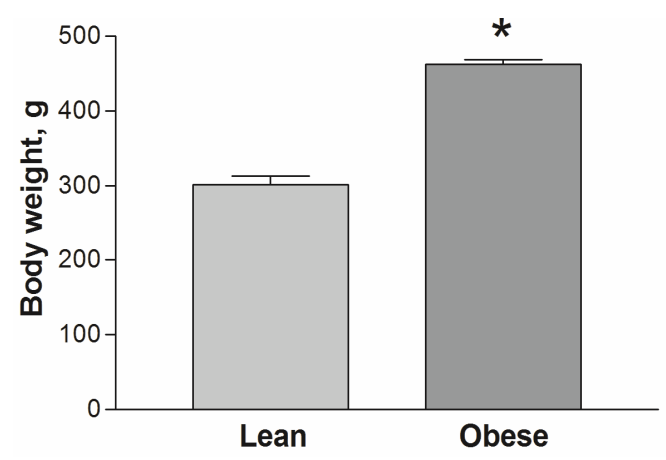

(a)
\#10500C, Camarillo, CA). Slides were rinsed with PBS and incubated with secondary goat polyclonal anti-rabbit antibody (1:1000 dilution, Abbiotec, LCC \#252237, San Diego, CA,) for 1 hour. Immunohistochemical reactivity was detected after reaction with diaminobenzadine (Sigma-Aldrich, St. Louis, MO) and immunostained sections counterstained with hematoxylin.

\subsection{Evaluation of Immunostaining}

IHC-stained slides were examined blindly by light microscopy at 200 -fold magnification. URAT1 positive stained tubules in cortex and medulla were counted in 5 randomly selected consecutive fields. In the cortex, proximal and distal immunostained tubules were counted and the proximal/distal tubules ratio calculated. The samples were also evaluated for diffuseness and intensity of staining [22].

\subsection{Statistical Analysis}

Results were compared between groups using independent samples T-test and paired samples T-test (for comparing results between cortex and medulla within groups) and expressed as the mean \pm SEM. The 0.05 level of probability was used as the criterion of significance.

\section{Results}

\subsection{Body and Kidney Weights, and Glucose and UA Levels Are Increased in Obese, Diabetic Zucker Rats}

As previously reported, at the age of 12 weeks the obese, diabetic Zucker rats had higher body weights (462.22 \pm 6.62 vs. $301.67 \pm 11.30 \mathrm{~g}, \mathrm{p}<0.05$ ) and kidney weights $(1.259 \pm 0.070$ vs. $1.010 \pm 0.068 \mathrm{~g}, \mathrm{p}<0.05)$ than the lean, non-diabetic counterparts (Figure 1) [21]. Obese, diabetic Zucker rats also had higher blood glucose (Figure 2(a)), but not plasma creatinine ( $0.446 \pm 0.077$ vs. $0.631 \pm$ $0.155 \mathrm{mg} / \mathrm{dl} \mathrm{p}>0.05$ ) than the lean counterparts. Plasma UA levels were elevated in the obese rats (Figure 2(b)).

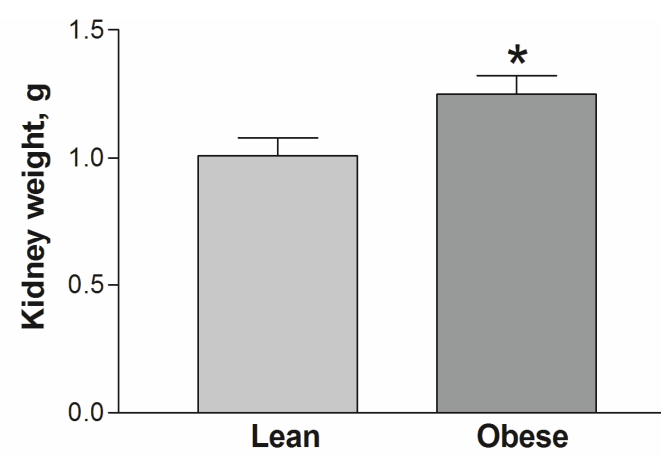

(b)

Figure 1. Body (a) and kidney weight (b) in lean and obese Zucker rats groups. ${ }^{*} \mathbf{p}<0.05$ between lean and obese rats, $\mathbf{n}=9$ both groups. All data are shown as mean \pm SEM. 


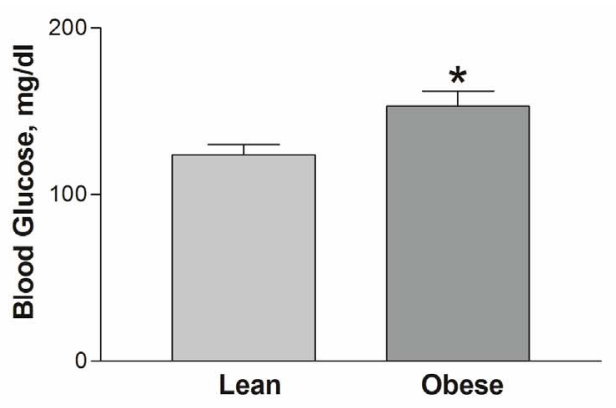

(a)

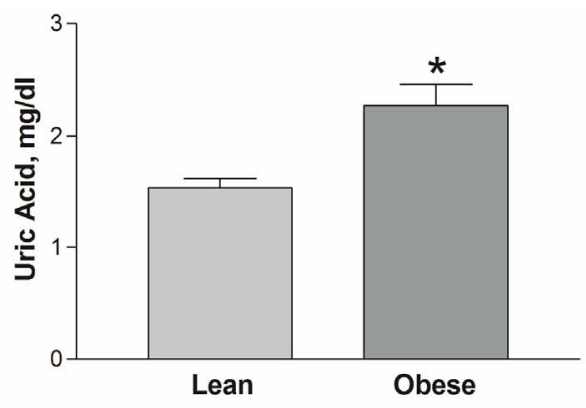

(b)

Figure 2. Glucose and UA Levels are increased in Obese, Diabetic Zucker Rats. At the age of 12 weeks the obese, diabetic Zucker rats had higher body weights $(462.22 \pm 6.62$ vs. $301.67 \pm 11.30 \mathrm{~g}, \mathrm{p}<0.05)$ and kidney weights $(1.259 \pm 0.070$ vs.1.010 \pm $0.068 \mathrm{~g}, \mathrm{p}<0.05$ ) than the lean, non-diabetic counterparts. Obese, diabetic Zucker rats also had higher blood glucose (a), but not plasma creatinine $(0.446 \pm 0.077$ vs. $0.631 \pm 0.155 \mathrm{mg} / \mathrm{dl} \mathrm{p}>\mathbf{0 . 0 5})$ than the lean counterparts. Plasma UA levels were elevated in the obese rats (b).

\subsection{URAT1 Is Expressed in the Kidney of Zucker Rats}

Western blot-URAT1 protein is expressed in both the kidney cortex (Figure 3(a)) and medulla (Figure 3(b)) of obese and lean Zucker rats. Western blot analysis of cortex revealed five URAT1 positive bands at 27, 31, 50, 62 and $70 \mathrm{kDa}$. A similar banding pattern was observed in Western blots of protein from the medulla. In addition, a $44 \mathrm{kDa}$ band was also detected in medulla. No differences were observed in URAT1 protein levels in the cortex of obese and lean Zucker rats. In medulla, however, the expression of the 44 and $50 \mathrm{kDa}$ proteins was significantly $(\mathrm{p}<0.05)$ higher in lean, non-diabetic Zucker rats. Within groups, the expression of the individual 27, 50, $62 \mathrm{kDa}$ URAT1 bands in cortex was two-fold higher than in medulla. Conversely, expression of the $70 \mathrm{kDa}$ URAT1 band was higher in medulla than in cortex.

Immunohistochemistry-URAT1 positive immunostaining was observed throughout the renal tubular system of both obese, diabetic and lean, non-diabetic Zucker rats (Figure 4). In kidney cortex, positive staining was observed equally in proximal and distal tubules without differences observed in diffuseness $(0.975 \pm 0.025$ vs. $0.920 \pm 0.053$ points, $\mathrm{p}>0.05)$, intensity of staining (1.000 \pm 0.037 vs. $0.950 \pm 0.063$ points, $p>0.05)$ or total IHC scores between diabetic and non-diabetic animals. URAT1 immunostaining was not detected in glomeruli (Figure 4(a)). In the medulla, the most intensive staining was observed in the inner medulla, associated with the collecting ducts. The same level of URAT1 positive staining was observed in the tubular system of cortex and medulla in both experimental groups.

\section{Discussion}

Diabetes is a risk factor for and the number one cause of chronic renal failure [23]. In addition, there is an associa- tion between elevated serum UA levels, gout, and kidney damage [6]. However, it has been debated over the years whether hyperuricemia is a cause or effect of renal dysfunction [24]. The most accepted hypothesis concerning the association between diabetes with gout is that there is a dominantly inherited defect in renal tubular urate handling, causing an imbalance in the reabsorption and secretion of urate by the kidney [25] and subsequent increases in blood UA levels [26]. URAT1 was the first UA transporter identified in the kidney and is thought to be the major mediator of the urate reabsorption pathway and the target of uricosuric drugs $[27,28]$. However a large number of additional transporters have been identified and these other reabsorbing (OAT 10, OAT 4 and GLUT9) and secretory (NPY1/NPT4, OAT 1 and OAT 3) transporters may also play a significant role [11]. As reported previously $[29,30]$, the obese, male, diabetic Zucker rats have higher blood UA levels than lean, nondiabetic counterparts. Western blots showed the presence of multiple URAT1 positive bands of 27, 31, 50, 62 and $70 \mathrm{kDa}$ in both cortex and medulla and an additional unique $44 \mathrm{kDa}$ band in medulla. Western blot analysis of URAT1 in mice demonstrated both 62 and $70 \mathrm{kDa}$ proteins. The $70 \mathrm{kDa}$ N-glycosylated form is found at the brush border membrane and the native $62 \mathrm{kDa}$ form is located on intracellular organelles [31]. Glycosylation plays critical roles in regulation of intracellular targeting, protein folding, modulation of biological activeity, maintenance of protein stability and formation of recognition structures for interactions with diverse external ligands [32,33]. Glycosylation is necessary to proper trafficking of the OATs family of proteins which includes transport of URAT1 to the plasma membrane and may enhance substrate recognition. Post-translational phosphorylation may also regulate the activity of transporters [33-35], and URAT1 has potential PKA and PKC phosphorylation sites [13]. URAT1 positive immunostaining in Zucker 

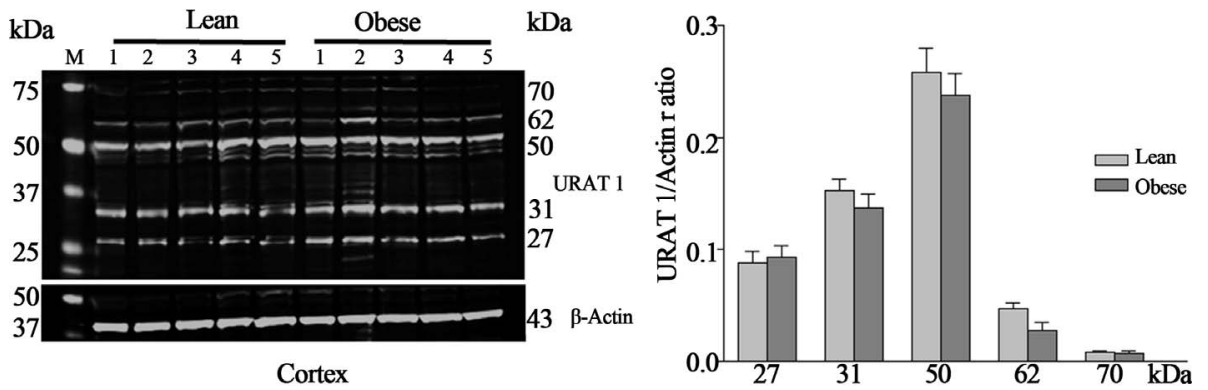

(a)
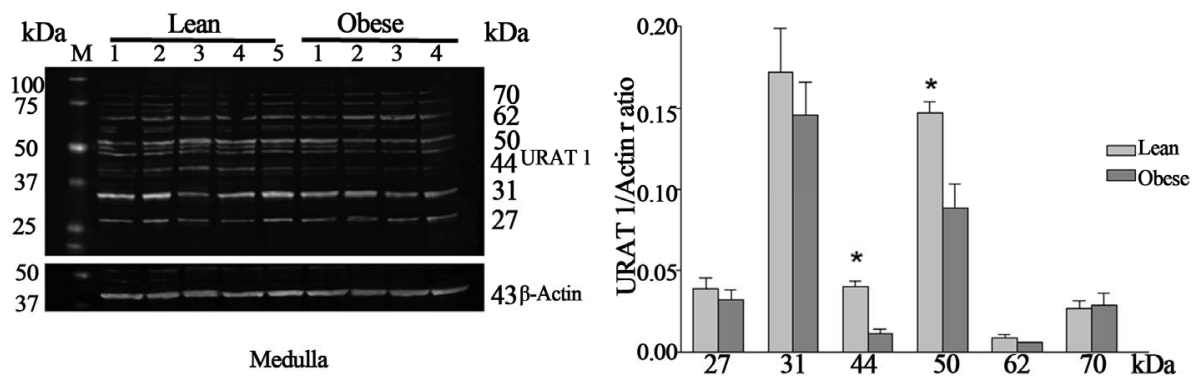

(b)

Figure 3. Representative Western blots of URAT1 and actin and URAT1/actin ratios from lean and obese Zucker rat kidney cortex (a) and medulla (b). URAT1 positive bands of 27, 31, 50 (three bands), 62 and $70 \mathrm{kDa}$ were observed in cortex and 27, 31, 50 (three bands), 44, 62 and $70 \mathrm{kDa}$ in medulla. ${ }^{*} \mathrm{p}<0.05$ between lean and obese rats, $\mathrm{n}=9$ both groups. $\mathrm{M}-$ molecular weight marker.

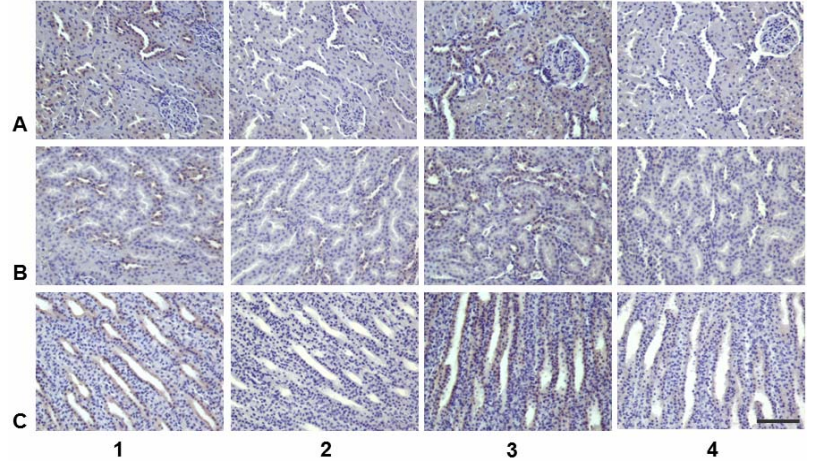

Figure 4. URAT1 positive immunostaining in kidney of lean $(1,2)$ and obese $(3,4)$ Zucker rats. A-cortex, B-outer medulla, C-inner medulla; 1 \& 3-anti-URAT1 antibody; $2 \& 4$-isotype control. Bar $=100 \mathrm{~nm}$.

rats was found in proximal and distal renal tubular segments in both cortex and medulla, but not in glomeruli, similar to the results reported in male Wistar rats [36]. Interestingly in mice [31] and humans [8], URAT1 has been described only in cortical tubules. Although Western blots of URAT1 in humans have shown a single 40 $\mathrm{kDa}$ protein, functional studies indicate that rat URAT1 mediates reabsorption of UA in a similar manner to human URAT1 and thus it may be possible to extrapolate findings in rats to uncover mechanisms in humans [36]. Based on molecular size, six distinct URAT1 proteins were found in Zucker rat kidney. In addition to post- translational modifications, the observed proteins may arise from translation of alternatively spliced mRNA transcripts which are now known to be characteristic of most genes [37]. Absolute and relative levels of the six transcripts differ between cortex and medulla. There are no increases in any transcript in the diabetic animals. In fact, absolute levels of the 44 and $50 \mathrm{kDa}$ proteins in medulla are decreased in the obese, diabetic rats compared to the lean rats. This may be a response to an increased filtered load of UA in the diabetic animals resulting in decreased secondary reabsorption, as alterations in splicing are known to occur due to internal environmental or epigenetic cues [37,38]. Alternatively, URAT1 protein levels may not accurately predict transporter activity. Others have shown a decrease in the 60 kDa URAT1 transcript in cortex with exercise and hyperuricemia in rats, associated with elevated URAT1 mediated urate reabsorption [39]. Alternatively spliced transcripts may also have non-coding regulatory functions [40]. It is also possible that URAT1 or a closely related protein is involved in the transport of other anions in the rat medulla $[11,12]$.

\section{Conclusion}

In male Zucker rats, URAT1 protein expression was observed in both kidney cortex and medulla. Uric acid elevation in the obese group was associated with decreases 
in the 44 and $50 \mathrm{kDa}$ URAT1 proteins in renal medulla.

\section{Authors Contributions}

WMW contributed the original idea for the study and participated in animal and biochemical protocols. WMW also received funding for this project with a grant from The Centers for Osteopathic Research and Education (CORE). SRI and KDM provided the laboratory space and supplies to perform the experiments. YS substantially participated in animal, biochemical and immunohistochemistry protocols, performed data analysis and prepared figures. AW participated in immunohistochemistry protocols and evaluation of samples. KDM performed Western blots, contributed to the original idea of the study, and participated in the analysis and interpretation of results. FVN assisted with data analysis and interpretation and preparation of the figures. RM provided expertise on immunohistochemistry. YS, FVN and SRI wrote the manuscript, KM edited the manuscript, and RM reviewed the immunohistochemistry sections of the manuscript.

\section{REFERENCES}

[1] R. Constantinescu and H. Zetterberg, "Urate as a Marker of Development and Progression in Parkinson's Disease,” Drugs Today, Vol. 47, No. 5, 2011, pp. 369-380.

[2] P. Fuhua, D. Xuhui, Z. Zhiyang, J. Ying, Y. Yu, T. Feng, L. Jia, G. Lijia and H. Xueqiang, "Antioxidant Status of Bilirubin and Uric Acid in Patients with Myasthenia Gravis," Neuroimmunomodulation, Vol. 19, No. 1, 2012, pp. 43-49. doi:10.1159/000327727

[3] A. Taniguchi and N. Kamatani, "Control of Renal Uric Acid Excretion and Gout," Current Opinion in Rheumatology, Vol. 20, No. 2, 2008, pp. 192-197. doi:10.1097/BOR.0b013e3282f33f87

[4] M. Hamburger, H. S. Baraf, T. C. Adamson, J. Basile, L. Bass, B. Cole, P. P. Doghramji, G. A. Guadagnoli, F. Hamburger and R. Harford, "2011 Recommendations for the Diagnosis and Management of Gout and Hyperuricemia," The Physician and Sportsmedicine, Vol. 39, No. 4, 2011, pp. 98-123. doi:10.3810/psm.2011.11.1946

[5] H. M. Lai, C. J. Chen, B. Y. Su, Y. C. Chen, S. F. Yu, J. H. Yen, M. C. Hsieh, T. T. Cheng and S. J. Chang, "Gout and Type 2 Diabetes Have a Mutual Inter-Dependent Effect on Genetic Risk Factors and Higher Incidences,” Rheumatology (Oxford), Vol. 51, No. 4, 2011, pp. 715-720.

[6] F. Viazzi, G. Leoncini and R. Pontremoli, "Cardiovascular and Renal Effects of Hyperuricaemia and Gout," Reumatismo, Vol. 63, No. 4, 2012, pp. 253-262. doi:10.4081/reumatismo.2011.253

[7] R. J. Johnson, M. A. Lanaspa and E. A. Gaucher, "Uric Acid: A Danger Signal from the RNA World that May Have a Role in the Epidemic of Obesity, Metabolic Syndrome, and Cardiorenal Disease: Evolutionary Considerations," Seminars in Nephrology, Vol. 31, No. 5, 2011, pp. 394-399. doi:10.1016/j.semnephrol.2011.08.002
[8] A. Enomoto, H. Kimura, A. Chairoungdua, Y. Shigeta, P. Jutabha, S. H. Cha, M. Hosoyamada, M. Takeda, T. Sekine and T. Igarashi, "Molecular Identification of a Renal Urate Anion Exchanger that Regulates Blood Urate Levels,” Nature, Vol. 417, No. 6887, 2002, pp. 447-452.

[9] T. Kimura, S. Amonpatumrat, A. Tsukada, T. Fukutomi, P. Jutabha, T. Thammapratip, E. J. Lee, K. Ichida, N. Anzai and H. Sakurai, "Increased Expression of SLC2A9 Decreases Urate Excretion from the Kidney,” Nucleosides Nucleotides Nucleic Acids, Vol. 30, No. 12, 2011, pp. 1295-1301. doi:10.1080/15257770.2011.628354

[10] K. Ichida, "What Lies behind Serum Urate Concentration? Insights from Genetic and Genomic Studies," Genome Medicine, Vol. 1, No. 12, 2009, p. 118. doi:10.1186/gm118

[11] A. So and B. Thorens, "Uric Acid Transport and Disease," Journal of Clinical Investigation, Vol. 120, No. 6, 2010, pp. 1791-1799. doi:10.1172/JCI42344

[12] N. Anzai and H. Endou, "Urate Transporters: An Evolving Field,” Seminars in Nephrology, Vol. 31, No. 5, 2011, pp. 400-409. doi:10.1016/j.semnephrol.2011.08.003

[13] M. A. Hediger, R. J. Johnson, H. Miyazaki and H. Endou, "Molecular Physiology of Urate Transport," Physiology (Bethesda), Vol. 20, No. 2, 2005, pp. 125-133.

[14] M. Doshi, Y. Takiue, H. Saito and M. Hosoyamada, “The Increased Protein Level of URAT1 Was Observed in Obesity/Metabolic Syndrome Model Mice,” Nucleosides Nucleotides Nucleic Acids, Vol. 30, No. 12, 2011, pp. 1290-1294. doi:10.1080/15257770.2011.603711

[15] Z. Miao, S. Yan, J. Wang, B. Wang, Y. Li, X. Xing, Y. Yuan, D. Meng, L. Wang and J. Gu, "Insulin Resistance Acts as an Independent Risk Factor Exacerbating HighPurine Diet Induced Renal Injury and Knee Joint Gouty Lesions," Inflammation Research, Vol. 58, No. 10, 2009, pp. 659-668. doi:10.1007/s00011-009-0031-9

[16] T. M. Coimbra, U. Janssen, H. J. Grone, T. Ostendorf, U. Kunter, H. Schmidt, G. Brabant and J. Floege, "Early Events Leading to Renal Injury in Obese Zucker (Fatty) Rats with Type II Diabetes,” Kidney International, Vol. 57, No. 1, 2000, pp. 167-182. doi:10.1046/j.1523-1755.2000.00836.x

[17] P. N. Chander, O. Gealekman, S. V. Brodsky, S. Elitok, A. Tojo, M. Crabtree, S. S. Gross and M. S. Goligorsky, "Nephropathy in Zucker Diabetic Fat Rat Is Associated with Oxidative and Nitrosative Stress: Prevention by Chronic Therapy with a Peroxynitrite Scavenger Ebselen," Journal of the American Society of Nephrology, Vol. 15, No. 9, 2004, pp. 2391-2403. doi:10.1097/01.ASN.0000135971.88164.2C

[18] E. Ionescu, J. F. Sauter and B. Jeanrenaud, “Abnormal Oral Glucose Tolerance in Genetically Obese $(\mathrm{Fa} / \mathrm{Fa})$ Rats," American Journal of Physiology, Vol. 248, No. 5, 1985, pp. 500-506.

[19] M. Hosoyamada, Y. Takiue, T. Shibasaki and H. Saito, "The Effect of Testosterone upon the Urate Reabsorptive Transport System in Mouse Kidney," Nucleosides $\mathrm{Nu}$ cleotides Nucleic Acids, Vol. 29, No. 7, 2010, pp. 574-579. doi:10.1080/15257770.2010.494651

[20] Y. Takiue, M. Hosoyamada, M. Kimura and H. Saito, “The Effect of Female Hormones upon Urate Transport Sys- 
tems in the Mouse Kidney," Nucleosides Nucleotides Nucleic Acids, Vol. 30, No. 2, 2011, pp. 113-119. doi:10.1080/15257770.2010.551645

[21] Y. Slyvka, S. R. Inman, R. Malgor, E. J. Jackson, J. Yee, O. Oshogwemoh, J. Adame and F. V. Nowak, "Protective Effects of Antioxidant-Fortified Diet on Renal Function and Metabolic Profile in Obese Zucker Rat," Endocrine, Vol. 35, No. 1, 2009, pp. 89-100. doi:10.1007/s12020-008-9121-7

[22] V. Tugcu, M. Bas, E. Ozbek, E. Kemahli, Y. V. Arinci, M. Tuhri, T. Altug and A. I. Tasci, "Pyrolidium Dithiocarbamate Prevents Shockwave Lithotripsy-Induced Renal Injury through Inhibition of Nuclear Factor-Kappa B and Inducible Nitric Oxide Synthase Activity in Rats," Journal of Endourology, Vol. 22, No. 3, 2008, pp. 559-566. doi:10.1089/end.2007.0295

[23] A. J. Collins, R. N. Foley, B. Chavers, D. Gilbertson, C. Herzog, K. Johansen, B. Kasiske, N. Kutner, J. Liu and W. Peter, "United States Renal Data System 2011 Annual Data Report: Atlas of Chronic Kidney Disease \& EndStage Renal Disease in the United States," American Journal of Kidney Diseases, 2012, Vol. 59, Suppl. 1, pp. $1-420$.

[24] T. Gibson, "Hyperuricemia, Gout and the Kidney," Current Opinion in Rheumatology, Vol. 24, No. 2, 2012, pp. 127-131. doi:10.1097/BOR.0b013e32834f049f

[25] S. A. Eraly, V. Vallon, T. Rieg, J. A. Gangoiti, W. R. Wikoff, G. Siuzdak, B. A. Barshop and S. K. Nigam, "Multiple Organic Anion Transporters Contribute to Net Renal Excretion of Uric Acid,” Physiological Genomics, Vol. 33, No. 2, 2008, pp. 180-192. doi:10.1152/physiolgenomics.00207.2007

[26] G. Calabrese, H. A. Simmonds, J. S. Cameron and P. M. Davies, "Precocious Familial Gout with Reduced Fractional Urate Clearance and Normal Purine Enzymes,” The Quarterly Journal of Medicine, Vol. 75, No. 277, 1990, pp. 441-450.

[27] N. Anzai, P. Jutabha, S. Amonpatumrat-Takahashi and H. Sakurai, "Recent Advances in Renal Urate Transport: Characterization of Candidate Transporters Indicated by Genome-Wide Association Studies," Clinical and Experimental Nephrology, Vol. 16, No. 1, 2012, pp. 89-95. doi:10.1007/s10157-011-0532-z

[28] H. J. Shin, M. Takeda, A. Enomoto, M. Fujimura, H. Miyazaki, N. Anzai and H. Endou, "Interactions of Urate Transporter URAT1 in Human Kidney with Uricosuric Drugs," Nephrology (Carlton), Vol. 16, No. 2, 2011, pp. 156-162. doi:10.1111/j.1440-1797.2010.01368.x

[29] E. T. de Lemos, F. Reis, S. Baptista, R. Pinto, B. Sepodes, H. Vala, P. Rocha-Pereira, G. C. da Silva, N. Teixeira and A. S. Silva, "Exercise Training Decreases Proinflammatory Profile in Zucker Diabetic (Type 2) Fatty Rats,” Nutrition, Vol. 25, No. 3, 2009, pp. 330-339.

doi:10.1016/j.nut.2008.08.014
[30] Y. Li, Y. Qi, M. S. Kim, K. Z. Xu, T. H. Huang, X. Rong, M. Murray and J. Yamahara, "Increased Renal Collagen Cross-Linking and Lipid Accumulation in Nephropathy of Zucker Diabetic Fatty Rats," Diabetes/Metabolism Research and Reviews, Vol. 24, No. 6, 2008, pp. 498-506. doi:10.1002/dmrr.874

[31] M. Hosoyamada, K. Ichida, A. Enomoto, T. Hosoya and H. Endou, "Function and Localization of Urate Transporter 1 in Mouse Kidney," Journal of the American Society of Nephrology, Vol. 15, No. 2, 2004, pp. 261-268. doi:10.1097/01.ASN.0000107560.80107.19

[32] F. Zhou and G. You, "Molecular Insights into the Structure-Function Relationship of Organizanion Transporters OATs,” Pharmaceutical Research, Vol. 24, No. 1, 2007, pp. 28-36. doi:10.1007/s11095-006-9144-9

[33] N. Anzai, Y. Kanai and H. Endou, “Organic Anion Transporter Family: Current Knowledge,” Journal of Pharmacological Sciences, Vol. 100, No. 5, 2006, pp. 411-426. doi:10.1254/jphs.CRJ06006X

[34] K. Kuze, P. Graves, A. Leahy, P. Wilson, H. Stuhlmann and G. You, "Heterologous Expression and Functional Characterization of a Mouse Renal Organic Anion Transporter in Mammalian Cells,” The Journal of Biological Chemistry, Vol. 274, No. 3, 1999, pp. 1519-1524. doi:10.1074/jbc.274.3.1519

[35] K. Tanaka, W. Xu, F. Zhou and G. You, "Role of Glycosylation in the Organic Anion Transporter OAT1," The Journal of Biological Chemistry, Vol. 79, No. 15, 2004, pp. 14961-14966. doi:10.1074/jbc.M400197200

[36] M. Sato, T. Wakayama, H. Mamada, Y. Shirasaka, T. Nakanishi and I. Tamai, "Identification and Functional Characterization of Uric Acid Transporter Urat1 (Slc22a12) in Rats," Biochimica et Biophysica Acta, Vol. 1808, No. 6, 2011, pp. 1441-1447. doi:10.1016/j.bbamem.2010.11.002

[37] A. Sanchez-Pla, F. Reverter, M. C. R. de Villa and M. Comabella, "Transcriptomics: mRNA and Alternative Splicing,” Journal of Neuroimmunology, Vol. 248, No. 1-2, 2012, pp. 23-31.

[38] M. Montes, S. Becerra, M. Sanchez-Alvarez and C. Sune, "Functional Coupling of Transcription and Splicing," Gene, Vol. 501, No. 2, 2012, pp. 104-117. doi:10.1016/j.gene.2012.04.006

[39] S. Cirrik, B. U. Yavuzer and G. Oner, "Exercise-Induced Changes in Renal Urat1 Activity and Expression in Rats," Renal Failure, Vol. 32, No. 7, 2010, pp. 855-862. doi:10.3109/0886022X.2010.494805

[40] C. N. Niland, C. R. Merry and A. M. Khalil, "Emerging Roles for Long Non-Coding RNAs in Cancer and Neurological Disorders,” Frontiers in Genetics, Vol. 3, No. 3, 2012, p. 25. 